\title{
Comparing Two Algorithms to Add Large Strains to SMALl-STRAin FE CODE
}

\author{
By Antonio Rodríguez-Ferran ${ }^{1}$ and Antonio Huerta, ${ }^{2}$ Member, ASCE
}

\begin{abstract}
Aastract: Two algorithms for the stress update (i.e., time integration of the constitutive equation) in largestrain solid mechanics are discussed, with particular emphasis on two issues: (1) The incremental objectivity; and (2) the implementation aspects. It is shown that both algorithms are incrementally objective (i.e., they treat rigid rotations properly) and that they can be employed to add large-strain capabilities to a small-strain finiteelement (FE) code in a simple way. A set of benchmark tests, consisting of simple large deformation paths, have been used to test and compare the two algorithms, both for elastic and plastic analyses. These tests evidence different time-integration accuracy for each algorithm. However, it is also shown that the algorithm that is less accurate in general gives exact results for shear-free deformation paths.
\end{abstract}

\section{INTRODUCTION}

Many problems of interest in physics and engineering are nonlinear (Bathe 1982; Crisfield 1991; Zienkiewicz and Taylor 1991). Two basic types of nonlinearity are encountered in solid mechanics: (1) Material nonlinearity; and (2) geometric nonlinearity. Nonlinear material behavior is often described by a rate-form constitutive equation, relating some measure of the rate of deformation to a rate of stress (Malvern 1969). In a large-strain context, the choice of a proper stress rate is a key point, because the principle of objectivity (Marsden and Hughes 1983; Hughes 1984) should be respected: the constitutive equation must be independent of the observer. This is only achieved when objective quantities are employed. The material derivative of stress is not an objective tensor (Malvern 1969; Bathe 1982) and, therefore, an alternative, objective stress rate is needed. The basic idea is that rigid rotations induce no straining of the material, and this fact must be properly captured by the stress rate. Some common objective stress rates are the Jaumann rate (Key and Krieg 1982; Hughes 1984), the Green-Naghdi rate (Hughes 1984; Healy and Dodds 1992) and the Truesdell rate (Pinsky et al. 1983). In these stress rates, rigid rotations are represented respectively by the spin tensor $\boldsymbol{\omega}$, the rate-of-rotation tensor $\mathbf{\Omega}$, and the deformation gradient $F$ (Malvern 1969).

The objectivity of the constitutive equations should also be respected by the numerical algorithms employed for their time integration. This requirement is referred to as incremental objectivity (Hughes and Winget 1980). The basic idea is that incremental rigid rotations induce no incremental straining of the material.

Several stress update algorithms (i.e., algorithms for the numerical time integration of the constitutive equations) can be found in the literature [see, for instance, Bathe et al. (1975), Hallquist (1979), Hughes and Winget (1980), Nagtegaal and de Jong (1981), Key and Krieg (1982), Pinsky et al. (1983), Rubinstein and Atluri (1983), Hughes (1984), Pegon and Guélin (1986), Cuitiño and Ortiz (1992), Healy and Dodds (1992), and Rashid and Thorne (1996)]. In many cases, however, employing these algorithms to add large-strain capabilities to a

${ }^{1}$ Assoc. Prof., Dept. de Matemática Aplicada III, E.T.S. de Ingenieros de Caminos, Universitat Politècnica de Catalunya, Campus Nord C-2, E08034, Barcelona, Spain.

${ }^{2}$ Prof., Dept. de Matemática Aplicada III, E.T.S. de Ingenieros de Caminos, Universitat Politècnica de Catalunya, Campus Nord C-2, E-08034, Barcelona, Spain. E-mail: huerta@etseccpb.upc.es small-strain finite-element (FE) code is a cumbersome task, because they require the computation of new quantities or the use of new concepts [such as multiplicative kinematics (Cuitiño and Ortiz 1992)], not employed for a small strain analysis.

If the Jaumann stress rate is used, for instance, incremental rigid rotations over the time step $\Delta t$ are represented by $\mathbf{Q}$ (Hughes and Winget 1980)

$$
\mathbf{Q}=\left(\mathbf{I}-\frac{1}{2} \Delta t \boldsymbol{\omega}\right)^{-1}\left(\mathbf{I}+\frac{1}{2} \Delta t \omega\right)
$$

In Key and Krieg (1982), $\mathbf{Q}$ is computed according to (1) and then half-angle trigonometric formulas are used to get the square root of $\mathbf{Q}$, which can be seen as the incremental rigid rotations over half a time step. This allows the computation of the stress increment in the midstep configuration $\left(t_{n+1 / 2}\right)$.

If (1) is used directly, it requires matrix inversion. This matrix inversion can be avoided by using the Cayley-Hamilton theorem (Hughes 1984), or by integrating numerically the generating matrix differential equation for $\mathbf{Q}$ (Rubinstein and Atluri 1983), $d Q / d t=\omega Q$.

For the Green-Naghdi rate, on the other hand, incremental rigid rotations are represented by the rotation tensor $\mathbf{R}$, arising from the polar decomposition $\mathbf{F}=\mathbf{R U}$ of the deformation gradient (Malvern 1969). Typically $\mathbf{R}$ must be computed twice per time step, at $t_{n+1 / 2}$ and $t_{n+1}$.

This can be done by using the Cayley-Hamilton theorem to compute $\mathbf{U}$ explicitly and then get $\mathbf{R}$ as $\mathbf{F U}^{-1}$ (Hughes 1984). To avoid inverting $\mathbf{U}, \mathbf{U}^{-1}$ is directly computed in Healy and Dodds (1992). To do this, the eigenvalues of $\mathbf{U}$ are obtained by means of a Jacobi transformation.

From the previous discussion, it is clear that the algorithms mentioned earlier require the computation of quantities that are not readily available in a small-strain FE code.

This paper discusses two incrementally objective algorithms, based on Truesdell stress rate, that allow transformation of an existing small-strain FE code into a large-strain code in a simple way (Rodríguez-Ferran and Huerta 1994). Only the particular case in which the elastic part of the deformation is modeled by a hypoelastic law - a common choice in nonlinear computational mechanics - will be addressed here. The first algorithm (Bathe et al. 1975) uses the full Lagrange strain tensor, including quadratic terms to account for large strains. The second algorithm, presented in Pinsky et al. (1983), employs the same strain tensor as in a small-strain analysis, but computed in the midstep configuration.

Various implementation aspects for the two algorithms are discussed. It is shown, in particular, that very few additional features must be added to a code with small-strain and nonlinear material behavior to enable its use for large-strain analysis.

The two algorithms are tested and compared with the help 
of a set of benchmark tests, consisting of simple deformation paths. Moreover, it is also shown that for some particular deformation paths, the first algorithm, which is less accurate in general, captures the exact solution, while the second algorithm does not.

This paper is organized as follows. Some preliminaries, including the basic equations of large-strain solid mechanics and the concept of objectivity, are briefly reviewed in Section 2 . The two stress update algorithms are presented in Section 3. After some introductory remarks, the notion of incremental objectivity is reviewed in Section 3.1. The two algorithms are then shown in Section 3.2, and their implementation in a small-strain FE code is discussed in Section 3.3. Section 4 deals with the numerical examples. Finally, some concluding remarks are made in Section 5.

\section{PRELIMINARIES}

\subsection{Basic Equations}

The first ingredient of continuum mechanics is the equation of motion, $\mathbf{x}=\mathbf{x}(\mathbf{X}, t)$, which yields the position $\mathbf{x}$ of material particles, denoted by their material coordinates $\mathbf{X}$, at time $t$ (Malvern 1969). If the initial spatial coordinates are employed as material coordinates, the material displacements can be defined as $u(t)=\mathbf{x}(t)-\mathbf{X}$. Once the displacements are defined, the starting point for strain representation is the deformation gradient $\mathbf{F}$
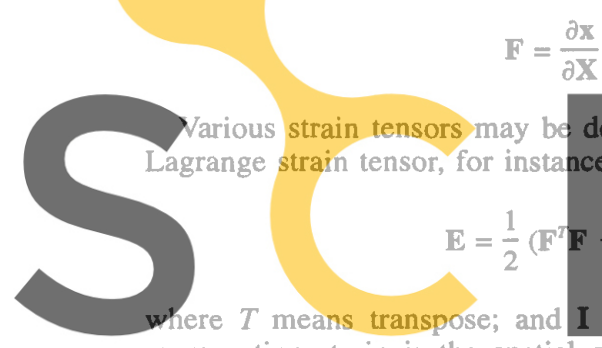

Various strain tensors may be defined by means of $\mathbf{F}$. The agrange strain tensor, for instance, is

$T$ means transpose; and $\mathbf{I}=$ identity. Another tensor the spatial gradient of velocity $\mathbb{\Perp}$. This censor yields relevant tensors if decomposed into symmetric

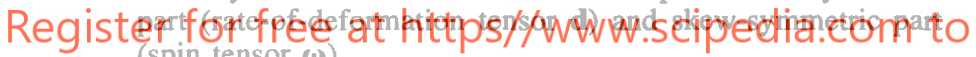
(spin tensor $\omega$ )

$$
\mathbf{l}=\frac{\partial \mathbf{v}}{\partial \mathbf{x}}=\mathbf{d}+\boldsymbol{\omega}
$$

In some problems, displacements and/or strains are large. Then the relation between displacements and strains is not linear and, moreover, the governing equations must be solved over the current configuration $\Omega_{t}$ at time $t$, not over the initial configuration $\Omega_{0}$. Since the motion that transforms $\Omega_{0}$ into $\Omega_{t}$ is precisely the fundamental unknown, a geometrically nonlinear problem is obtained.

The balance laws of continuum mechanics state the conservation of mass, momentum, and energy (Malvern 1969). For a wide range of problems in solid mechanics, three simplifying assumptions are common: (1) Mechanical and thermal effects are uncoupled; (2) the density is constant; and (3) inertia forces are negligible in comparison to the other forces acting on the body (quasi-static process). The mechanical problem is then governed by the momentum balance alone, which becomes a static equilibrium equation

$$
\frac{\partial \boldsymbol{\sigma}_{i j}}{\partial x_{j}}+\mathbf{b}_{i}=0
$$

where $\sigma=$ Cauchy stress tensor; and $\mathbf{b}=$ body force. Eq. (5) models many problems of practical interest -including, for instance, various forming processes ("Simulation" 1995).

\subsection{Stress Tensors}

The most common representation of stress is the Cauchy stress tensor $\sigma$, defined in the current configuration $\Omega_{t}$ and already presented in (5). This tensor has a clear physical meaning, because it involves only forces and surfaces in the current configuration.

In a large-strain context, other representations of stress are possible and indeed useful. The key idea (Pinsky et al. 1983) is that $\Omega_{0}$ and $\Omega_{t}$ are different configurations, so tensors defined in each configuration cannot be combined by operations, such as subtraction and addition. Let ${ }^{\circ} \sigma$ and ' $\sigma$ be the Cauchy stress tensors at the initial time $t_{0}$ and current time $t$, respectively; the increment of stress may not be defined as ' $\sigma-{ }^{0} \sigma$, because the two tensors are referred to different configurations. As stress increments will be needed to update stresses, a proper definition is required.

An alternative representation of stress is the second PiolaKirchhoff tensor $\mathbf{S}$, defined as the pull-back of $\boldsymbol{\sigma}$

$$
\mathbf{S}=J \mathbf{F}^{-1} \mathbf{\sigma} \mathbf{F}^{-r}
$$

where $J=\operatorname{det}(\mathbb{F})=$ Jacobian of the motion, which reflects the variation of unit volume associated to the deformation, and the inverse of the deformation gradient $\mathbf{F}^{-1}$ is employed to transform $\sigma$ from $\Omega_{t}$ to $\Omega_{0}$. Eq. (6) is called the pull-back Piola transformation. It must be remarked that $\mathrm{S}$ represents the state of stress at time $t$, but referred to configuration $\Omega_{0}$, and should not be confused with ${ }^{\circ} \sigma$, the stress at initial time $t_{0}$.

Eq. (6) may be reversed, and then $\sigma$ may be seen as the push-forward of $\mathbf{S}$

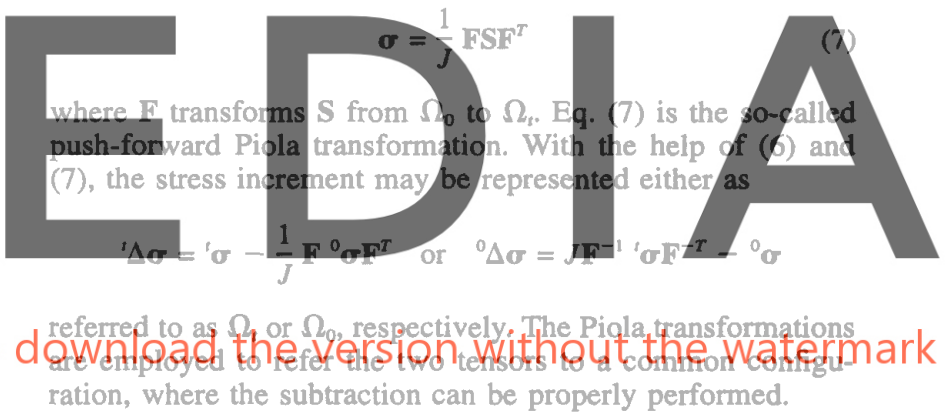

\subsection{Constitutive Equations and Objectivity}

In nonlinear solid mechanics, the material behavior is often described by a rate-form constitutive equation, relating the stress rate to velocity and/or its derivatives and the stress state (and eventually, some internal variables). The particular case of hypoelastic materials, where the stress rate depends linearly on the rate-of-deformation tensor d (Malvern 1969) will be considered here to present the two stress update algorithms. The two algorithms, however, can be extended to elastoplastic problems, by profiting from the decomposition of the rate-ofdeformation $\mathbf{d}$ into elastic and plastic parts (Khan and Huang 1995). The hypoelastic constitutive law is

$$
\dot{\text { o }}=\mathbf{C}: \mathbf{d}
$$

where $\dot{\boldsymbol{\sigma}}=$ material rate of stress; and $\mathbf{C}=$ elastic moduli tensor (Malvern 1969).

In fact, (8) is only valid for small strains. As shown next, the material rate of stress $\dot{\sigma}$ may not be employed to represent stress variation in a large-strain problem, because it is not an objective tensor.

The principle of objectivity is a fundamental requirement regarding the constitutive equation in large-strain solid mechanics (Marsden and Hughes 1983; Hughes 1984): if the constitutive equations really describe the physical behavior of the 
continuum, they must be independent of the observer. This requirement is fulfilled if objective quantities appear in the constitutive equations. A quantity is said to be objective if it transforms in a proper tensorial manner under a superposed rigid-body motion. Let the rigid motion be represented by an orthogonal rotation tensor $\mathbf{Q},\left(\mathbf{Q}^{-1}=\mathbf{Q}^{T}\right)$ and a translation $\mathbf{a}$. The time-dependent relation between old and new coordinates is then $\mathbf{x}^{\text {new }}(t)=\mathbf{Q}(t) \mathbf{x}+\mathbf{a}(t)$. It is postulated that the Cauchy stress $\sigma$ is objective. As it is a second-order tensor, it transforms according to $\boldsymbol{\sigma}^{\text {new }}(t)=\mathbf{Q}(t) \boldsymbol{\sigma}(t) \mathbf{Q}(t)^{T}$. By derivating this expression with respect to time, it can be checked that the material derivative of an objective tensor is not objective. This invalidates the use of $\dot{\boldsymbol{\sigma}}$ as the stress rate in a rate-form constitutive equation. An alternative, objective stress rate $\sigma^{*}$ is therefore needed.

As for the rate-of-deformation tensor d, it can be shown that it is an objective tensor, so it may be employed to represent strains in a constitutive equation. Indeed, the hypoelastic constitutive equation is rewritten, in a large strain framework as

$$
\sigma^{*}=\mathrm{C}: \mathrm{d}
$$

The stress rate $\sigma^{*}$ is not uniquely determined by the objectivity principle. Some classical options reviewed in Pinsky et al. (1983) are the Jaumann, the Green-Naghdi, and the Truesdell rates

$$
\sigma_{T}^{*}=\dot{\sigma}-1 \sigma-\sigma l^{T}+\operatorname{tr}(d) \sigma
$$

where $\operatorname{tr}(\mathbf{d})=$ trace of tensor $\mathbf{d}$. It can be easily checked that the terms in the right-hand side of (10) in addition to the ma-

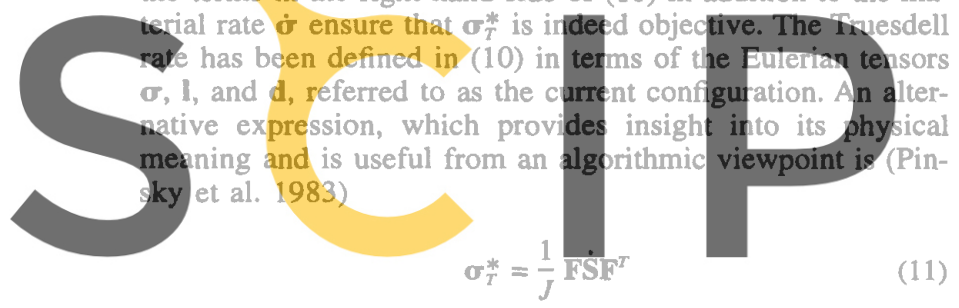

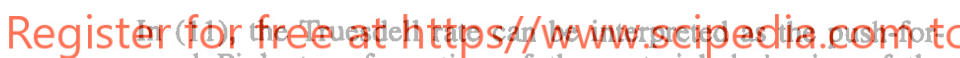
ward Piola transformation of the material derivative of the second Piola-Kirchhoff stress sensor $\mathbf{S}$. Thus, instead of using the time derivative of the Cauchy stress tensor that yields the nonobjective material rate, the Truesdell rate is preferred because it is by construction an objective rate. In (11) it can be seen that the Truesdell rate proceeds in three steps: (1) $\sigma$ is pulled back into $\mathbf{S}$; (2) the material derivative of $\mathbf{S}$ is performed; and (3) the resulting rate is pushed forward into the current configuration. Where the rationale is that the material derivative of a material tensor (i.e., a tensor referred to as the initial configuration) yields an objective tensor.

\section{TWO STRESS UPDATE ALGORITHMS FOR LARGE STRAINS}

If the FE method (Hughes 1987; Zienkiewicz and Taylor 1991 ) is employed, the partial differential equation (5) is transformed into the nonlinear systems of equations.

$$
\mathbf{r}(\mathbf{u})=\mathbf{f}_{\text {int }}(\mathbf{u})-\mathbf{f}_{\text {ext }}(\mathbf{u})=\mathbf{0}
$$

where $\mathbf{f}_{\mathrm{int}}=$ internal force vector; $\mathbf{f}_{\mathrm{ext}}=$ external load vector; and $\mathbf{r}=$ residual forces. Eq. (12) is typically solved incrementally with a displacement-based implicit method (Bathe 1982; Crisfield 1991). The fundamental unknowns are then the in cremental displacements $\Delta \mathbf{u}={ }^{n+1} \mathbf{x}-{ }^{n} \mathbf{x}$ from one (known) equilibrium configuration $\Omega_{n}$ at time $t_{n}$ to a new (unknown) equilibrium configuration $\Omega_{n+1}$ at time $t_{n+1}=t_{n}+\Delta t$. These incremental displacements are first predicted and then iteratively corrected. Within each iteration, the constitutive equation must be integrated over the time increment to update the stresses and check the equilibrium.

\subsection{Incremental Objectivity}

In the context of the incremental buildup of the solution, it is useful to define the incremental versions of the tensors presented in (2) and (3). Let ${ }^{n} F$ and ${ }^{n+1} F$ be the deformation gradients relating $\Omega_{n}$ and $\Omega_{n+1}$, respectively, to the reference configuration $\Omega_{0}$ (see Fig. 1). The incremental deformation gradient ${ }^{n} \boldsymbol{\Lambda}$ is

$$
{ }^{n} \boldsymbol{\Lambda}={ }^{n+1} \mathbf{F}{ }^{n} \mathbf{F}^{-1}
$$

which refers configuration $\Omega_{n+1}$ to configuration $\Omega_{n}$. The corresponding incremental Lagrange strain tensor is then

$$
{ }^{n} \underline{\Delta} \mathbf{E}=\frac{1}{2}\left({ }^{n} \Lambda^{T}{ }^{n} \mathbf{\Lambda}-\underline{I}\right)
$$

Incremental objectivity is a requirement on the algorithm for the numerical time integration of the constitutive equation, which is often presented as the discrete counterpart of the principle of objectivity (Hughes and Winget 1980). Let the incremental deformation gradient ${ }^{n} \Lambda$ relating configurations $\Omega_{n}$ and $\Omega_{n+1}$ be an orthogonal tensor ${ }^{n} \mathbf{R}$. The numerical algorithm is said to be incrementally objective if it predicts a stress state at $t_{n+1}$ that is simply a rotation of the stress state at $t_{n}$

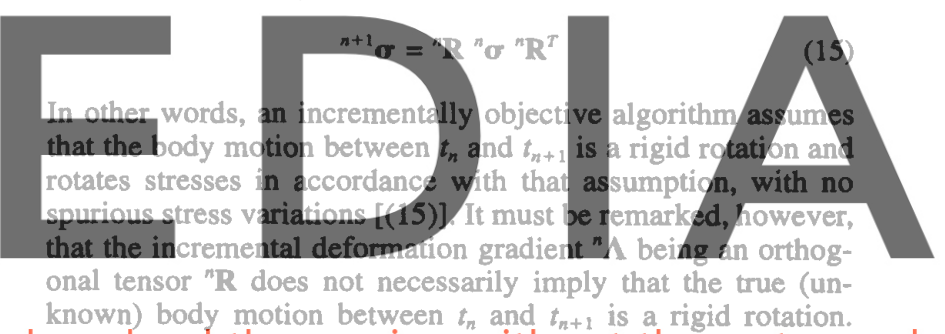

\section{known) body motion between $t_{n}$ and $t_{n+1}$ is a rigid rotation.}

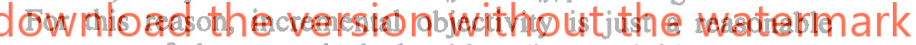
property of the numerical algorithm (i.e., a rigid rotation is assumed when possible) rather than a physical requirement like the principle of objectivity (Rodriguez-Feman and Huerta 1994; Rodríguez et al. 1997).

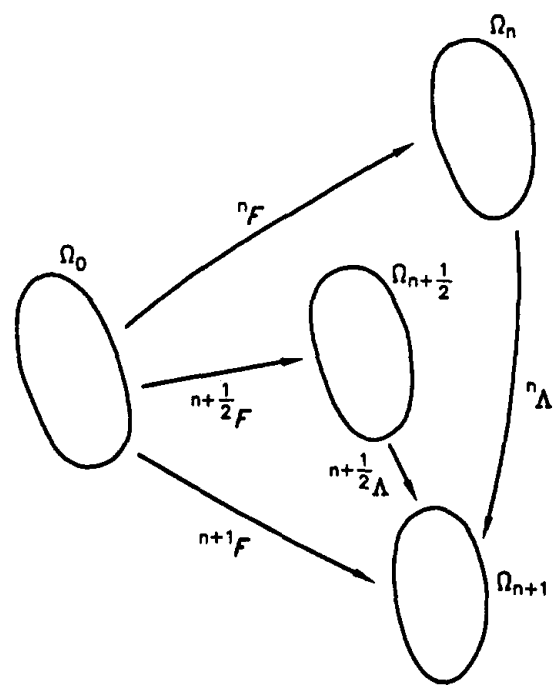

FIG. 1. Doformation Gradients in Incremental Analysie 


\subsection{Two Stress Update Algorithms for Large Stains}

\subsubsection{First Stress Update Algorithm}

It is possible to employ the incremental Lagrange strain tensor defined in (14) as the strain measure in the increment $\Delta t$ (Bathe et al. 1975). The stress increment is then

$$
{ }^{n} \Delta \sigma=C::^{n} \Delta \mathbf{E}
$$

where the superscript $n$ in $\Delta \sigma$ indicates that this tensorial quantity is, as ${ }^{n} \Delta E$, referred to configuration $\Omega_{n}$.

In a large-strain context it is no longer valid to compute the new stresses ${ }^{n+1} \boldsymbol{\sigma}$ by simply adding the stress increment ${ }^{n} \Delta \sigma$ to the old stresses " $\sigma$, because these latter two tensors are in the configuration $\Omega_{n}$, and ${ }^{n+1} \sigma$ is sought in the configuration $\Omega_{n+1}$. It is necessary to transform the tensors adequately by means of the push-forward Piola transformation [(7)]. The numerical algorithm for the stress update is then

$$
{ }^{n+1} \boldsymbol{\sigma}={ }^{n} J^{-1}{ }^{n} \Lambda^{n} \boldsymbol{\sigma}{ }^{n} \Lambda^{T}+{ }^{n} J^{-1 n} \Lambda^{n} \Delta \sigma{ }^{n} \Lambda^{T}
$$

where the Jacobian ${ }^{n} J=\operatorname{det}\left({ }^{n} \Lambda\right)$; and the incremental deformation gradient, [(13)] is employed to push-forward both " $\sigma$ and ${ }^{n} \Delta \sigma$ into the new configuration $\Omega_{n+1}$.

This algorithm is incrementally objective: if ${ }^{n} \boldsymbol{\Lambda}$ is an orthogonal tensor, (14) yields a null strain tensor ${ }^{n} \Delta \mathbf{E}$, and (16) reduces to (15), thus predicting a rigid rotation of stresses, with no spurious stress variations. Note that the use of the full incremental Lagrange tensor, including quadratic terms, is essential for the incremental objectivity of the algorithm.

\subsubsection{Second Stress Update Algorithm}

An alternative, more accurate numerical algorithm will be shown next. Following Pinsky et al. (1983), the hypoelastic constitutive equation is written in terms of the Truesdell objective stress rate $[(10)]$,

$$
\sigma_{T}^{*}=\mathbf{C}: \mathbf{d}
$$

A basic ingredient of this algorithm is that $\mathbf{d}$ is evaluated in the midstep configuration $\Omega_{n+1 / 2}$, defined through linear inter-

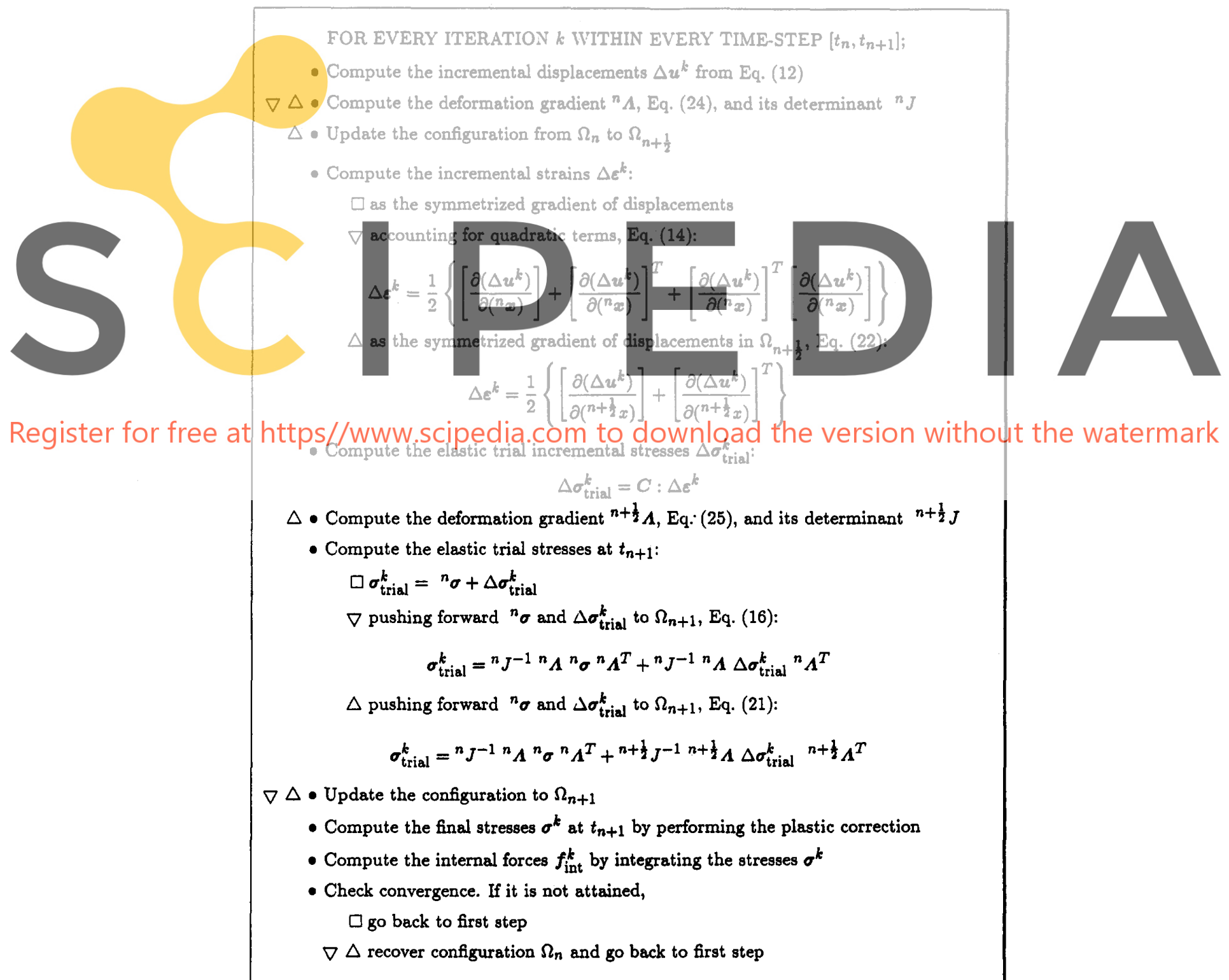

FiG. 2. Materially Nonlinear Analysis: $\square$, Small Stralns; $\nabla$, Large Strains, First Algorlthm; $\Delta$, Large Strains, Second Algorithm 
polation between $\Omega_{n}$ and $\Omega_{n+1}$. The midstep spatial coordinates are

$$
{ }^{n+1 / 2} \mathrm{X}=\frac{1}{2}\left({ }^{n} \mathrm{X}+{ }^{n+1} \mathrm{x}\right)={ }^{n} \mathrm{X}+\frac{1}{2} \Delta \mathrm{u}
$$

the associated deformation gradient is

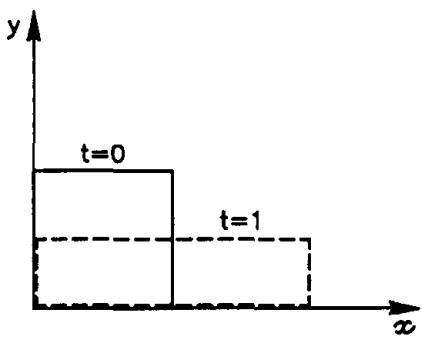

FIG. 3. Extension and Compression Test-Problem Statement

\section{Elastic}

SIGMAXX/E

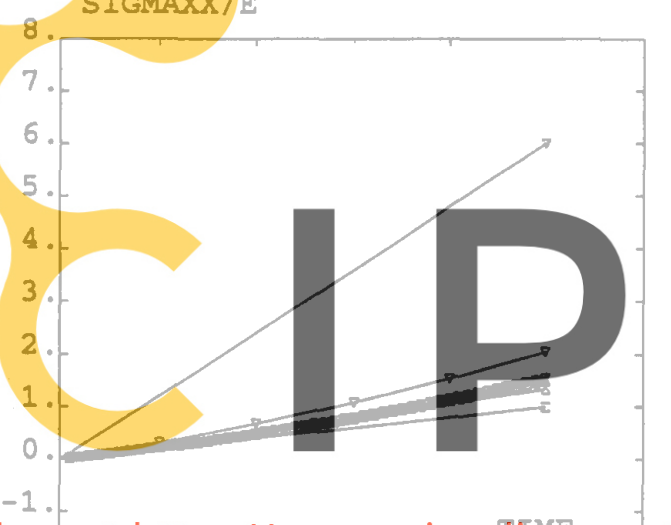

Register for free at https//www.scipedra.eom (a)

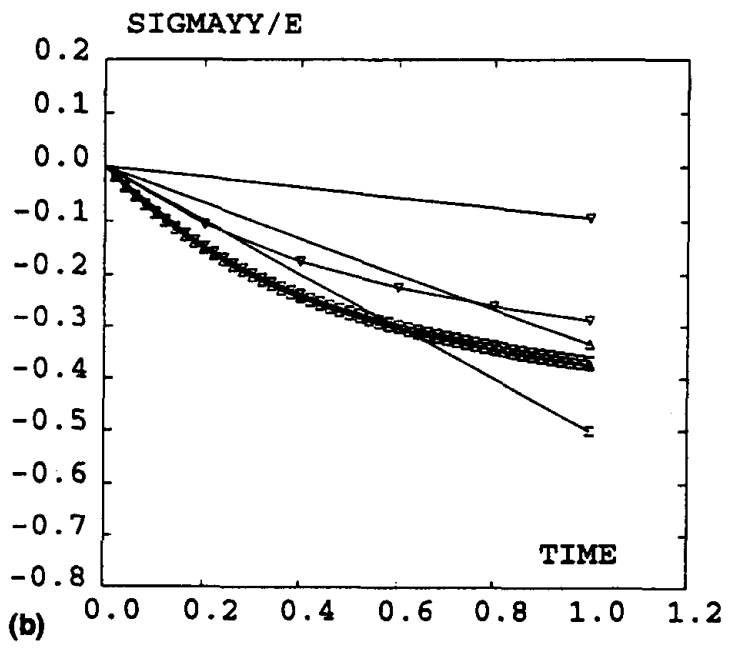

$$
{ }^{n+1 / 2} \mathbf{F}=\frac{1}{2}\left({ }^{n} \mathbf{F}+{ }^{n+1} \mathbf{F}\right)
$$

and the incremental deformation gradient relating the midstep and final configurations is

$$
{ }^{n+1 / 2} \boldsymbol{\Lambda}={ }^{n+1} \mathbf{F}^{n+1 / 2} \mathbf{F}^{-1}
$$

The different deformation gradient tensors are summarized in Fig. 1. Using a midpoint rule algorithm to integrate (17), the stress update becomes

${ }^{n+1} \sigma={ }^{n} J^{-1 n} \Lambda^{n} \sigma^{n} \Lambda^{T}+\left.{ }^{n+1 / 2} J^{-1 n+1 / 2} \Lambda(\Delta t C: d)\right|_{n+1 / 2}{ }^{n+1 / 2} \Lambda^{T}$

with the Jacobian ${ }^{n+1 / 2} J$ defined as $\operatorname{det}\left({ }^{n+1 / 2} \Lambda\right)$. As in (16), tensors referred to as the initial and midstep configurations are pushed forward into the final one by means of the appropriate incremental deformation gradients ${ }^{n} \Lambda$ and ${ }^{n+1 / 2} \Lambda$.

Recalling the definition of $\mathbf{d}[(4)]$, the approximation to ${ }^{n+1 / 2} \mathbf{d}$ needed in (21) will be

\section{Plastic}

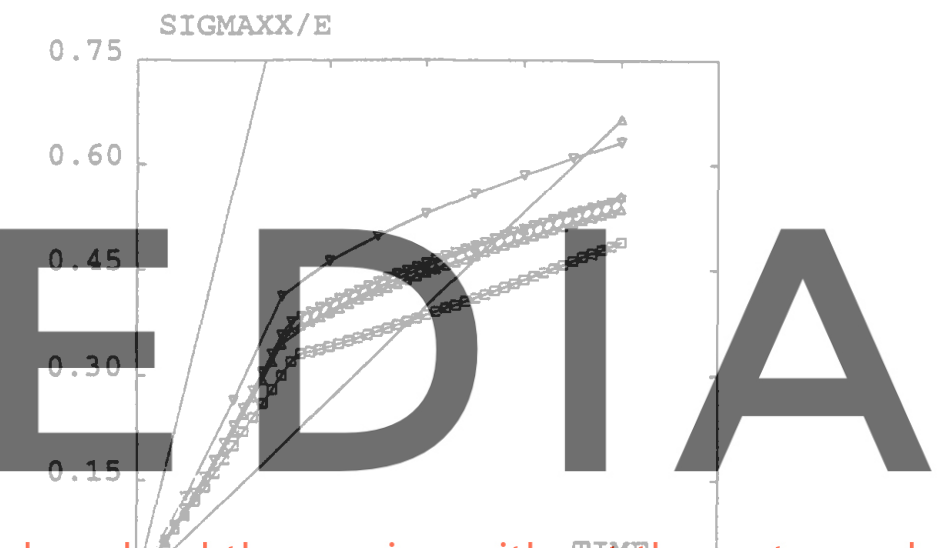

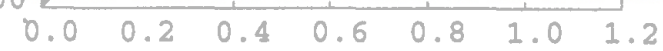

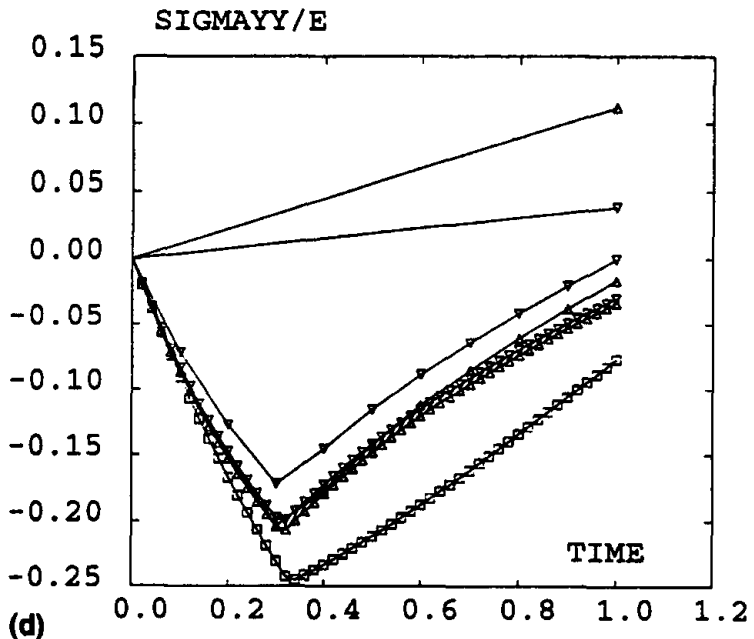

\section{$\nabla$ First \\ $\triangle$ Second \\ $\square$ Small strain}

FIG. 4. Extension and Compression Test; Stress versus TIme Curves Computed with Two Algorlthms and Small-Strain Analysis. Elastic Analysis: (a) $\sigma_{x x} ;$ (b) $\sigma_{p}$. Plastic Analysis: (c) $\sigma_{x x ;}$ (d) $\sigma_{y}$ 


$$
{ }^{n+1 / 2} \mathbf{d}=\frac{{ }^{n+1 / 2} \Delta \varepsilon}{\Delta t}=\frac{1}{2 \Delta t}\left\{\left[\frac{\partial(\Delta \mathbf{u})}{\partial\left({ }^{n+1 / 2} \mathbf{x}\right)}\right]+\left[\frac{\partial(\Delta \mathbf{u})}{\partial\left(^{n+1 / 2} \mathbf{x}\right)}\right]^{T}\right\}
$$

The stress increment is then

$$
{ }^{n+1 / 2} \Delta \boldsymbol{\sigma}=\left.(\Delta t \mathbf{C}: \mathbf{d})\right|_{n+1 / 2}=\mathbf{C}: \frac{1}{2}\left\{\left[\frac{\partial(\Delta \mathbf{u})}{\partial\left(^{n+1 / 2} \mathbf{x}\right)}\right]+\left[\frac{\partial(\Delta \mathbf{u})}{\partial{\left({ }^{n+1 / 2} \mathbf{x}\right)}^{T}}\right]^{T}\right\}
$$

It must be noted that in (22) the strain increment ${ }^{n+1 / 2} \Delta \varepsilon$ is represented by the symmetrized gradient of the incremental displacements, as in a small-strain analysis. No additional quadratic terms are needed in (23), because large strains are properly modeled by employing the midstep configuration to compute the gradient of displacements. As discussed in Pinsky et al. (1983), this algorithm is also incrementally objective. Moreover, the numerical tests of Section 4 show that its numerical performance (in terms of accuracy) is superior to that of the first algorithm. An accuracy analysis of the two algorithms, showing that the first one is first-order accurate in time and the second one is second-order accurate, can be found in Rodríguez-Ferran et al. (1997),

The two stress update algorithms can also be employed in elastoplasticity. The basic idea is to model the elastic part of the deformation with a hypoelastic law, and use any of the two algorithms to compute the elastic trial stress (Pinsky et al. 1983; Hughes 1984). After that, a plastic corrector - a radial return algorithm, for instance - is required to account for material nonlinearity (Hughes 1984).

\subsection{Implementation Aspects}

It is shown in this section that algorithms can be employed to add small-strain FE code in a simple way. The basic idea is that the incremental deformation gradients required in (16) and 21) can be computed in a straightorward manner by using quantities that are available in a small-strain code. Consider, for instance, the incremental deformation gradient " $\Lambda$ relating
$\Omega_{n}$ to $\Omega_{n+1}[(13)]$. Recalling the definition of $F$ in (2) and the

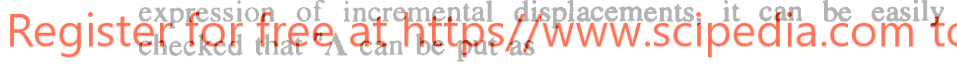

$$
{ }^{n} \boldsymbol{\Lambda}=\frac{\left.\partial{ }^{n+1} \mathbf{x}\right)}{\partial\left({ }^{(\mathbf{x})}\right.}=\mathbb{I}+\frac{\partial(\Delta \mathrm{u})}{\partial\left({ }^{(} \mathbf{x}\right)}
$$

If an updated Lagrangian formulation is used (Bathe 1982), the configuration $\Omega_{n}$ is taken as a reference to compute the incremental displacements. In such a context, " $\Lambda$ can be computed from (24) with the aid of standard nodal shape functions, by expressing $\Delta \mathbf{u}$ in terms of the nodal values of incremental displacements. Since the derivatives of shape functions are available in a standard FE code (Hughes 1987; Zienkiewicz and Taylor 1991), no new quantities must be computed to obtain ${ }^{n} \Lambda$.

As for ${ }^{n+1 / 2} \Lambda$, combining (18)-(20) renders

$$
{ }^{n+1 / 2} \Lambda=\frac{\partial\left({ }^{n+1} \mathbf{x}\right)}{\partial\left({ }^{n+1 / 2} \mathbf{x}\right)}=\mathbf{I}+\frac{1}{2} \frac{\partial(\Delta \mathbf{u})}{\partial\left(^{n+1 / 2} \mathbf{x}\right)}
$$

so ${ }^{n+1 / 2} \boldsymbol{\Lambda}$ can also be directly computed with the aid of the shape functions, once the configuration of the mesh has been updated from $\Omega_{n}$ to $\Omega_{n+1 / 2}$.

As a result, the only two additional features that are required to handle large strains are (1) the updating of mesh configuration; and (2) the computation of incremental gradient gradients [(24) and (25)]. This can be seen in Fig. 2, which compares the schematic algorithms for materially nonlinear analysis with small strains ( $\square$ ) to the large strain versions ( $\nabla$ first algorithm; $\Delta$ second algorithm).
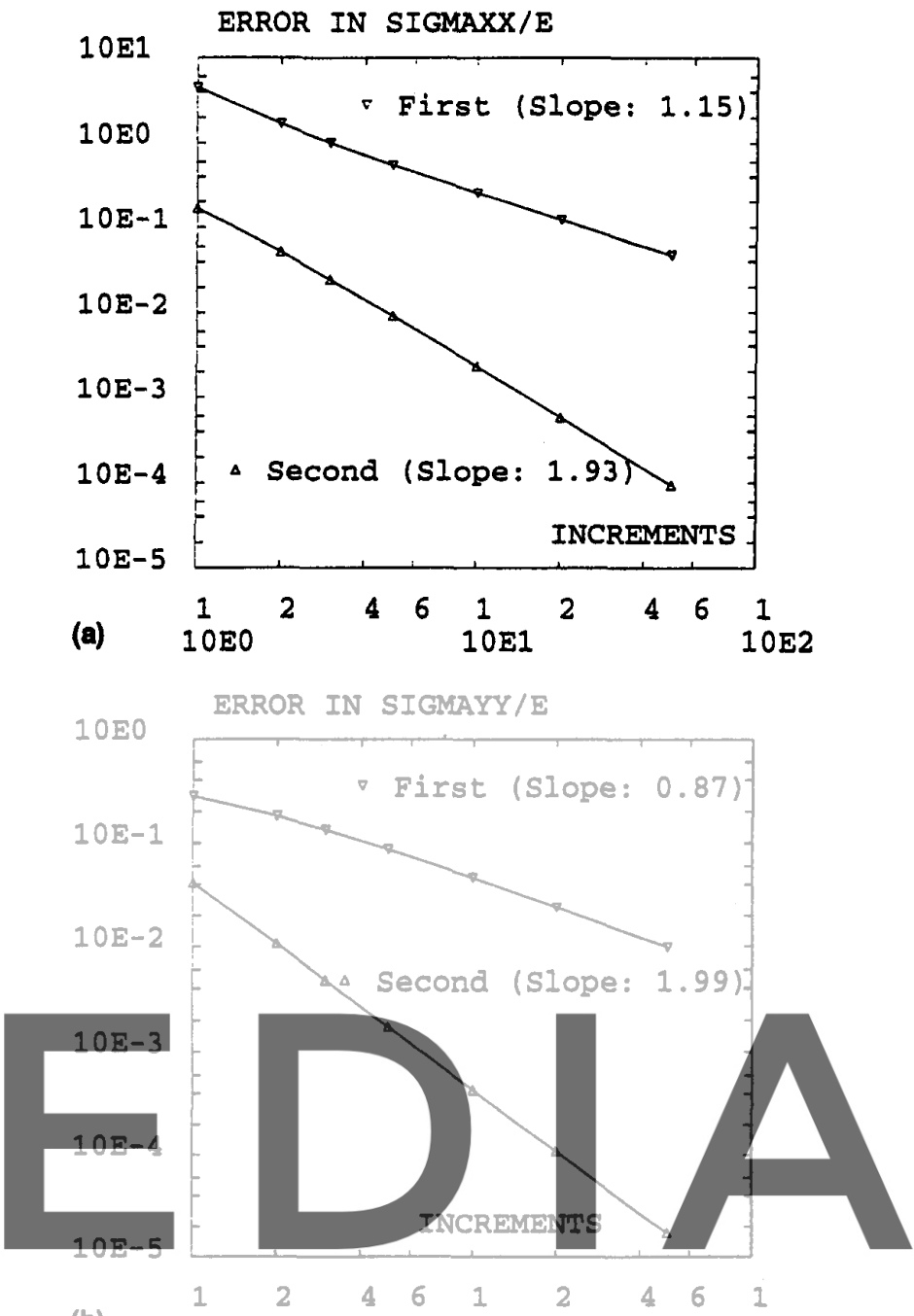

dowhloadthe version without the watermark

FIG. 5. Extenslon and Compression Test, Elestlc Analysis. Error In Final Value of Strese $(t=1)$ vereus Number of Time Steps: (a) $\sigma_{\text {mi }}(\mathrm{b}) \sigma_{\text {, }}$

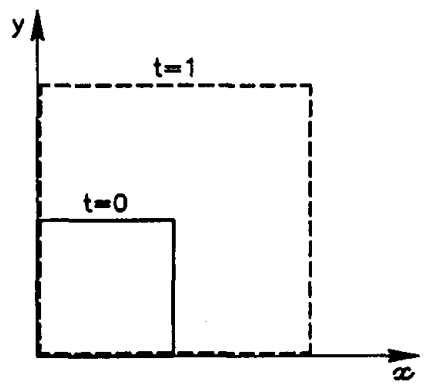

FIG. 6. Dllatation Test-Problem Statement

\section{NUMERICAL TESTS}

The two stress update algorithms presented in Section 3.2 have been compared with the help of various simple, largestrain, deformation paths: simple shear, uniaxial extension, extension and compression, dilatation, extension, and rotation. For brevity, only the last three will be shown in detail here. Both elastic and elastoplastic cases are considered. Elastic behavior will be represented by Young's modulus $E$ and a null Poisson's coefficient $\nu=0$. For the plastic cases, the consti- 
Elastic

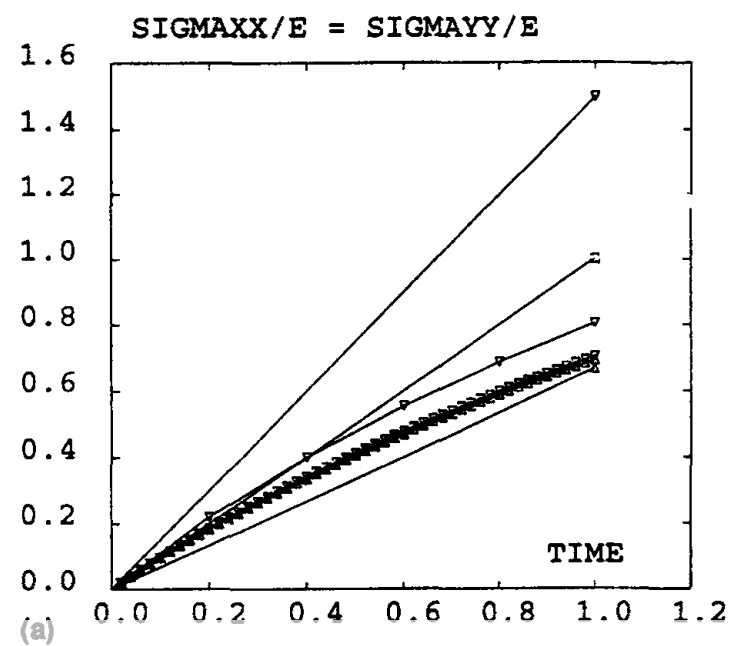

\section{Plastic}

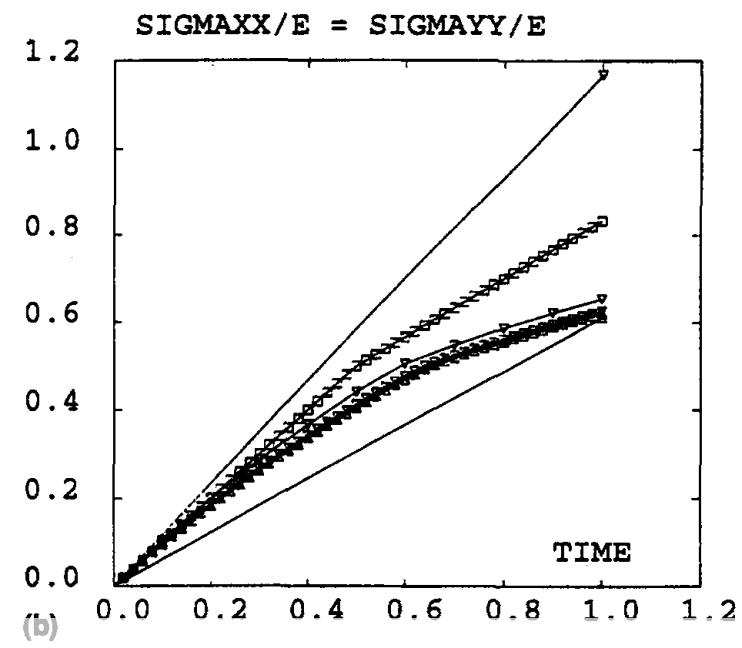

$\nabla$ First

$\triangle$ Second

Small strain

FIG. 7. Dilatation Test; Normal Stress versus Time Curves Computed with Two Algorithms and Small-Strain Analysis: (a) Elastlc Analysis; (b) Plastic Analysis

10E0

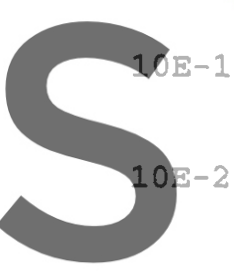

$10 \mathrm{E}-3$

\section{ERROR IN SIGMA/E}

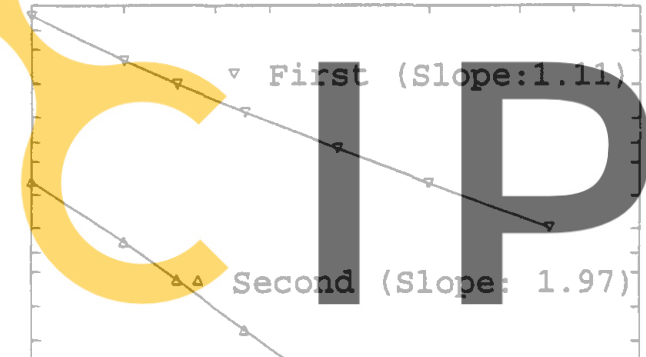

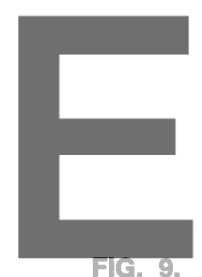

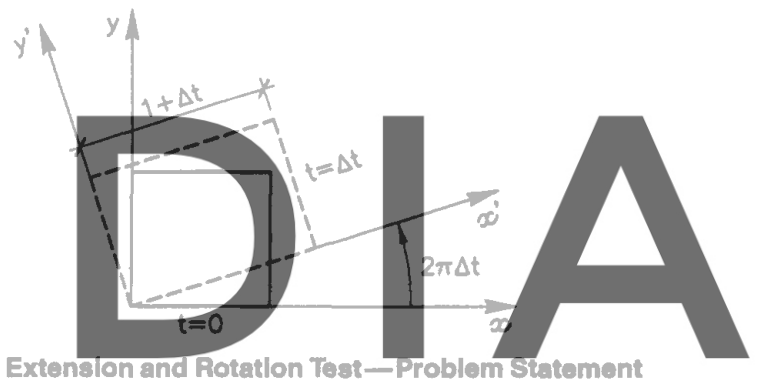

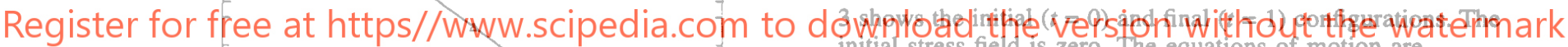

$10 \mathrm{E}-4$

$10 \mathrm{E}-5$

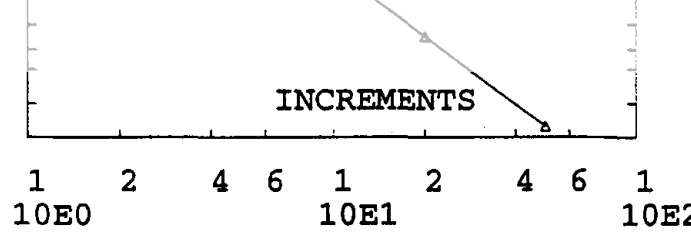

FIG. 8. Dilatation Test, Elastic Analysis. Error In Final Value of Normal Stress $(t=1)$ versus Numer of Time Steps

tutive law is assumed to be bilinear, with a plastic modulus of $E_{p}=E / 100$ and a yield stress of $\sigma_{y}=E / 2$.

The first algorithm is first-order accurate in time, while the second one is second-order accurate [see the proof in Rodríguez-Ferran et al. (1997).]. As a consequence, the general behavior is that results depend heavily on the number of time increments if the first algorithm is employed, and only slightly with the second one. However, the first algorithm shows a superior performance for some stress components in various tests, as shown in Section 4.3 and explained in detail in Rodríguez-Ferran and Huerta (unpublished work, 1998). For comparison purposes, a small-strain analysis is also performed.

\subsection{Extension and Compression}

A unit square undergoes extension in the $x$-direction and compression in the $y$-direction, with no change in volume. Fig.

$$
x(t)=X(1+t) ; \quad y(t)=Y /(1+t)
$$

If the Truesdell objective rate is employed [(17)] yields the system of ordinary differential equations

$(1+t) \dot{\sigma}_{x x}-2 \sigma_{x x}=\frac{E}{1+\nu} ; \quad \dot{\sigma}_{x y}=0 ; \quad(1+t) \dot{\sigma}_{y y}+2 \sigma_{y y}=-\frac{E}{1+\nu}$

which can be complemented with null initial conditions and solved to provide the analytical solution

$$
\sigma_{x r}(t)=E\left(t+\frac{t^{2}}{2}\right) ; \quad \sigma_{x y}(t)=0 ; \quad \sigma_{y y}(t)=\frac{E}{2}\left[\frac{1}{(1+t)^{2}}-1\right]
$$

Both algorithms have been employed, with different values of the time step $(1,2,3,5,10,20$, and 50 increments), to integrate the constitutive equation for the given deformation path, [(26)] from $t=0$ to $t=1$.

Fig. 4 presents the results for the elastic case $(1,5$, and 50 increments). The two stress update algorithms are capable of predicting a null $\sigma_{x y}$, but differences appear for $\sigma_{x x}$ and $\sigma_{y y}$ [Figs. 4(a and b)]. It can be seen that the first algorithm grossly overestimates stresses when not enough increments are employed and demands a small time step to get close to the analytical solution. The second algorithm, on the other hand, yields more accurate solutions, even if only one time step is 
Elastic
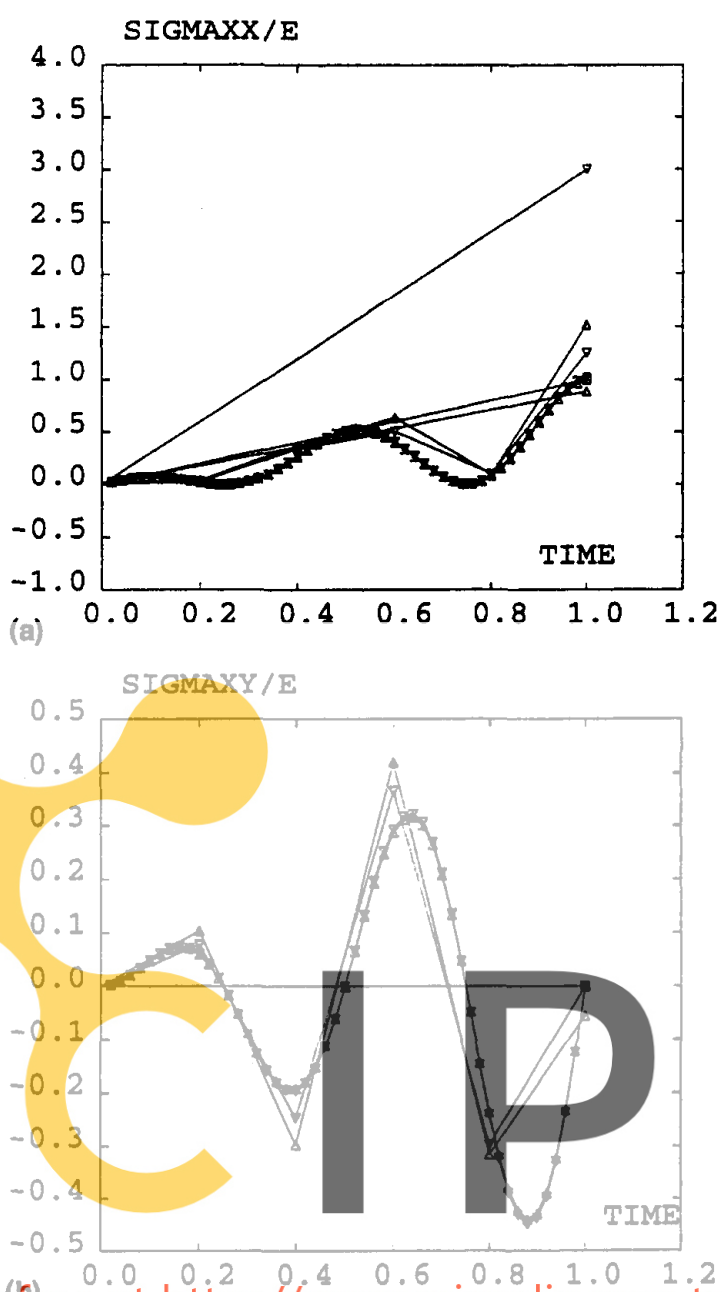

Register forlpree at https//WwW.scipedia.com to

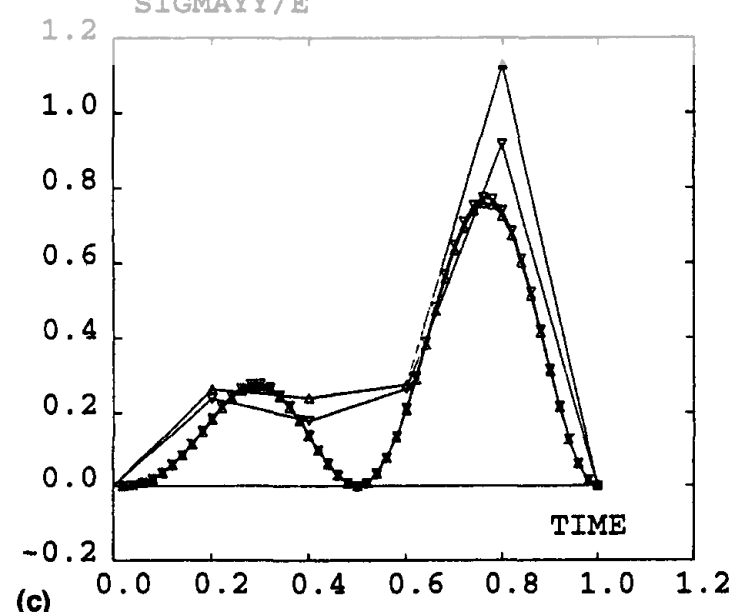

Plastic
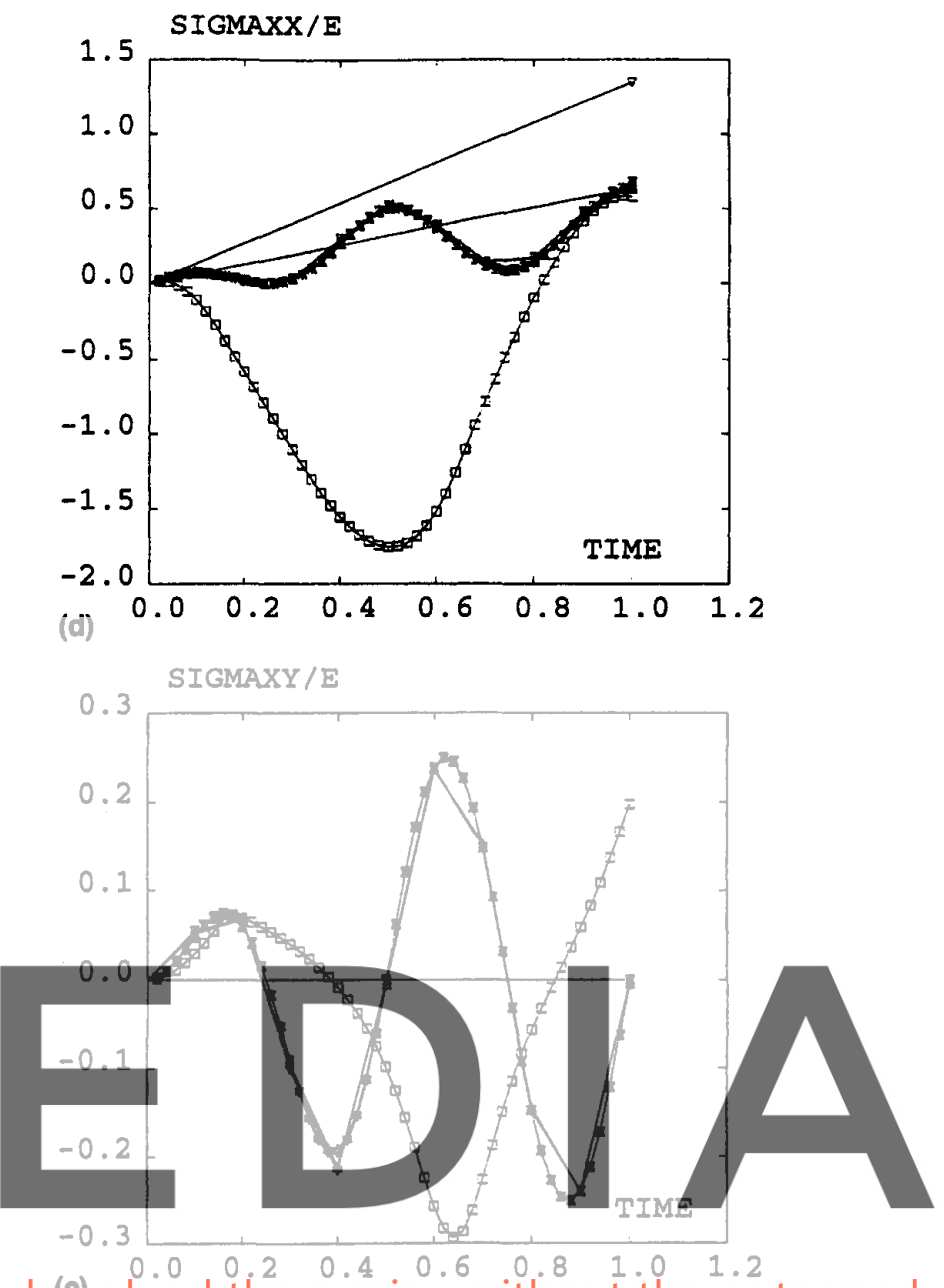

$\nabla$ First $\quad \triangle$ Second $\quad \square$ Small strain

FIG. 10. Extension and Rotation Test. Stress versus Time Curves Computed with Two Algorithms and Small-Strain Analysis. Elastlc Analysis: (a) $\sigma_{x x}$; (b) $\sigma_{x y} ;$ (c) $\sigma_{y}$. Plastlc Analysis: (d) $\sigma_{x x} ;\left(\right.$ (e) $\sigma_{x y} ;(b) \sigma_{y y}$ 
used. The output of a small strain analysis ( 1 increment), is also presented in Fig. 4; the obtained stresses differ significantly from the large-strain analyses, because the small-strain analysis only captures the linear terms of the analytical solution $[(27)]$.

Since the analytical solution is known, the error in the final stress $(t=1)$ can be computed and plotted versus the number of time increments (Fig. 5). In a log-log scale, a straight line with a slope equal to the order of the algorithm is expected, i.e., 1 for the first algorithm and 2 for the second [(21)]. For the two relevant components of stress, the observed slopes are close to the expected values: 1.15 (first algorithm) and 1.93 (second algorithm) for $\sigma_{x x}$ [Fig. 5(a)] and 0.87 (first algorithm) and 1.99 (second algorithm) for $\sigma_{y y}$ [Fig. 5(b)].

This test has also been carried out in the elastoplastic case $(1,10$, and 50 increments) [Fig. 4(c and d)]. As in the elastic problem, the first algorithm grossly overestimates the final stress if only one increment is employed, while the second one predicts much more accurate values. With a higher number of time steps, both algorithms converge to the same response. A small strain analysis, this time with 50 increments to account for material nonlinearity, is again not satisfactory. The need for a large-strain analysis is again demonstrated.

\subsection{Dilatation}

A unit square undergoes biaxial extension (Fig. 6). The equations of motion are $x(t)=X(1+t) ; y(t)=Y(1+t)$, and the analytical solution of (17) is $\sigma_{x x}(t)=\sigma_{y y}(t)=E \ln (1+t)$; $\sigma_{x y}(t)=0$. Both algorithms yield qualitatively correct results, in the sense that $\sigma_{x y}$ is zero and $\sigma_{x x}=\sigma_{y y}$ for any number of time steps and in both elastic and plastid modes. There are sharp differences, however, concerning convergence behtvion For the elastic case, for instance, the second algorithm prokides a better prediction with one time increment than the first one with five [Fig. 7(a)]. It can be seen in Fig. 8 that again, he algorithms behave as expected. The computed slopes are in this case 1.11 for the first algorithm and 1.97 for the second one. A similar comparison is valid in the plastic test, where one increment with the second algorithm gets closer to the

\subsection{Extension and Rotation}

In this last deformation path, a unit square undergoes a uniaxial extension and a superposed rigid rotation (Fig. 9). The equations of motion are

$$
\begin{aligned}
& x(t)=X(1+t) \cos (2 \pi t)-Y \sin (2 \pi t) ; \quad y(t)=X(1+t) \sin (2 \pi t) \\
& \quad+Y \cos (2 \pi t)
\end{aligned}
$$

and the analytical solution of (17) is

$\sigma_{x x}(t)=E t \cos ^{2}(2 \pi t) ; \quad \sigma_{x y}(t)=E t \sin ^{2}(2 \pi t) ;$

$$
\sigma_{y y}(t)=E t \sin (2 \pi t) \cos (2 \pi t)
$$

The output of the elastic analysis can be seen in Fig. 10. If only one increment is employed, the rotation part of the motion is not captured and the predicted stress is identical to that of a uniaxial extension test (Rodríguez-Ferran et al. 1997). With a higher number of steps, the comparative performance of the two algorithms is different from that of the previous tests. For the stress components $\sigma_{x y}$ and $\sigma_{y y}$, the first algorithm is the one that predicts correct null values for any number of time increments. In fact, this result illustrates a more general behavior. A unified treatment of shear-free deformation paths can be found in Rodriguez-Ferran and Huerta (unpublished work, 1998). In this reference it was shown that the first al-
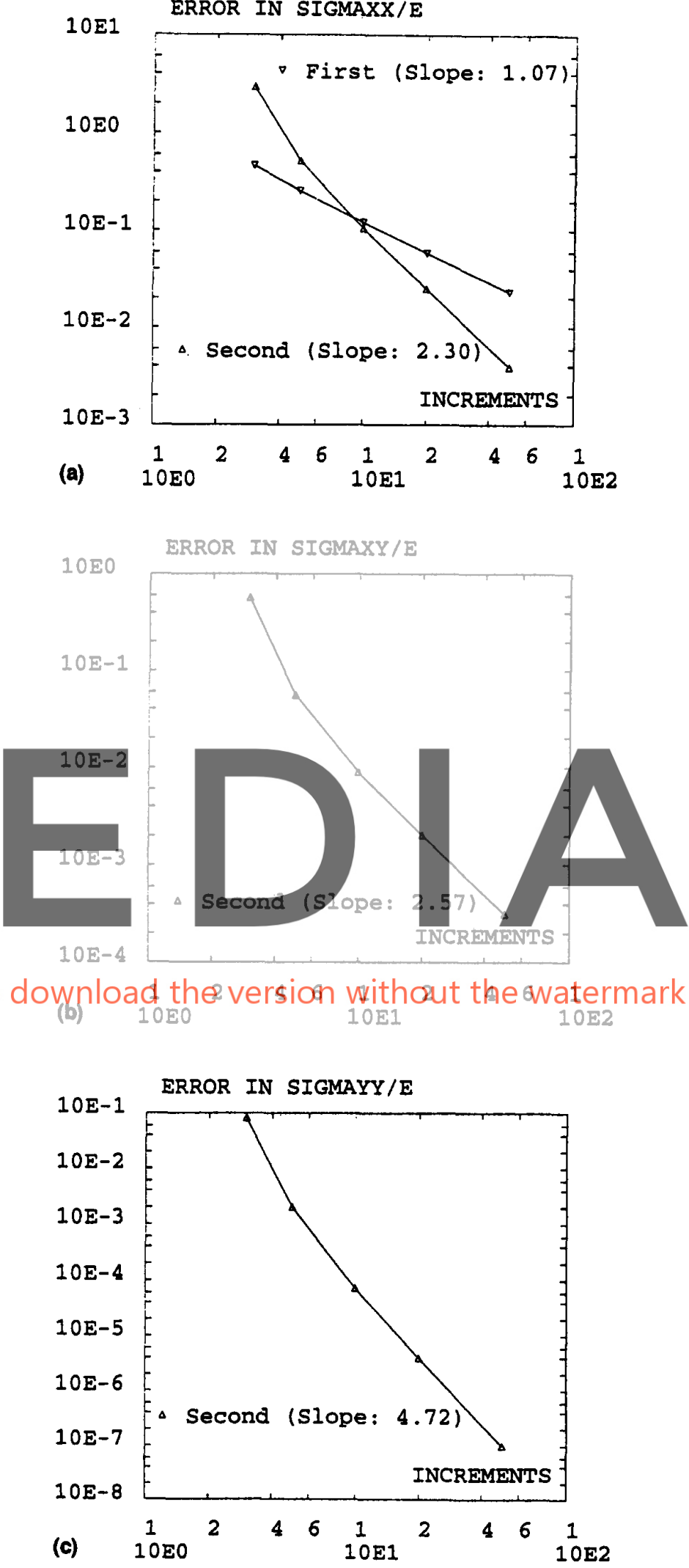

FIG. 11. Extension and Rotation Tost, Elastic Analysis. Error in Final Value of Stress $(t=1)$ versus Number of Time Steps; (a) $\sigma_{x x i}(b) \sigma_{x y} ;(c) \sigma_{y y}$ 
TABLE 1. Observed Order of Convergence for Varlous Tests

\begin{tabular}{l|c|c|c|c|c|c}
\hline \hline \multirow{2}{*}{$(1)$} & \multicolumn{3}{|c|}{ OBSERVED ORDER OF CONVERGENCE } \\
\cline { 2 - 7 } & \multicolumn{2}{|c|}{$\sigma_{x x}$} & \multicolumn{2}{|c|}{$\sigma_{x y}$} & \multicolumn{2}{c|}{$\sigma_{y y}$} \\
\cline { 2 - 7 } & $\nabla$ & $\Delta$ & $\nabla$ & $\Delta$ & $\nabla$ & $\Delta$ \\
& $(2)$ & $(3)$ & $(4)$ & $(5)$ & $(6)$ & $(7)$ \\
\hline Extension and compression & 1.15 & 1.93 & Exact & Exact & 0.87 & 1.99 \\
Dilatation & 1.11 & 1.97 & Exact & Exact & 1.11 & 1.97 \\
Simple shear & 1.09 & Exact & 1.16 & Exact & 1.00 & Exact \\
Uniaxial extension & 1.13 & 1.95 & Exact & Exact & Exact & Exact \\
Extension and rotation & 1.07 & 2.30 & Exact & 2.57 & Exact & 4.72 \\
\hline
\end{tabular}

gorithm correctly predicted null shear stresses for any number of time steps, while the second one did not.

As for the stress component $\sigma_{x x}$, the first algorithm performs better if a reduced number of time steps (five) is employed, and a larger number is required for the second algorithm to produce more accurate results. This behavior is illustrated by Fig. 11(a), where the error curves for $\sigma_{x x}$ of the two algorithms intersect each other. Again, the observed order of both schemes (1.07 for the first one and 2.30 for the second one) is in accordance with the expected values. Figs. 11(b and c) show the convergence behavior of the second algorithm for the other two stress components. It can be seen that the convergence to the exact analytical value is very fast, especially for $\sigma_{y y}$ [Fig. $11(\mathrm{c})]$

The outcome of the plastic analysis is also depicted in Fig. 10. Once again, a small-strain analysis with nonlinear material behavior turns out to be completely unsatisfactory, providing a solution that is qualitatively different from that of a large-

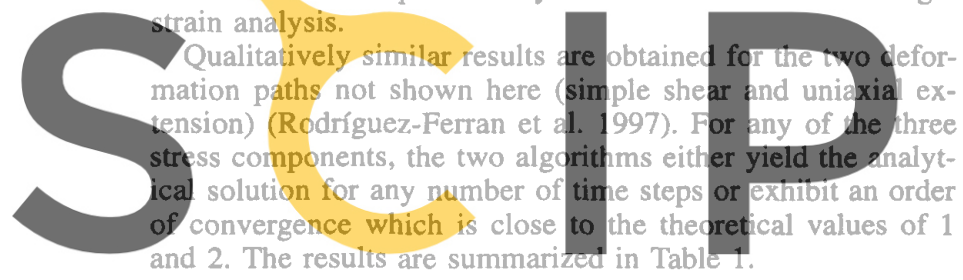

Register for free dt http SAKS Ww.scipedia.com to
Two numerical stress update algorithms for large strains have been discussed. The first one (Bathe et al. 1975) uses the full incremental Lagrange strain tensor, including quadratic terms, as the strain measure. The second one (Pinsky et al. 1983) works in the midstep configuration, where the symmetrized gradient of the displacement increment can be employed as the strain measure, as in a small-strain analysis.

Any of the two algorithms may be employed to enhance a small-strain FE code into a large-stain code in a simple way. In particular, it has been shown that if the code already handles material nonlinearity, adding large strains only involves two additional features: (1) Updating of the mesh configuration; and (2) computation of incremental deformation gradients, which are readily available from standard shape functions.

These two algorithms have been compared with the aid of a set of simple deformation paths, both in elasticity and elastoplasticity. The results show that the second algorithm is generally more accurate than the first one, the main reason being the use of the midstep configuration as a reference. In fact, it can be shown that the first algorithm is first-order accurate, and the second one is second-order accurate (Rodriguez-Ferran et al. 1997). Accuracy, however, is not the only relevant point: for shear-free deformation paths, there is no error in the shear component of the stress tensor computed with the first algorithm (Rodríguez-Ferran and Huerta, unpublished work, 1998). In the numerical tests, the two algorithms behave in very good agreement with their expected behavior. Analytical solutions are provided for the elastic analyses, which can be employed to verify the implementation of these (or any other) stress update algorithms.

\section{ACKNOWLEDGMENTS}

The writers wish to thank graduate student A. Vila for carrying out the various numerical tests. The writers also want to express their appreciation to Prof. A. Millard for fruitful discussions on the topic of large strains. The financial support from DGICYT PB94-1200 and the Human Capital and Mobility ERB-4050-PL-92-2002 is gratefully acknowledged.

\section{APPENDIX. REFERENCES}

Bathe, K. J. (1982). Finite procedures in engineering analysis. PrenticeHall, Inc., Englewood Cliffs, N.J.

Bathe, K. J., Ramm, E., and Wilson, E. L. (1975). "Finite element formulations for large deformation dynamic analysis." Int. J. Numer. Methods in Engrg., 9, 353-386.

Crisfield, M. A. (1991). Non-linear finite element analysis of solids and structures. John Wiley \& Sons, Inc., England.

Cuitiño, A., and Ortiz, M. (1992). "A material-independent method for extending stress update algorithms from small-strain plasticity to finite plasticity with multiplicative kinematics." Engrg. Computations, 9, $437-451$

Hallquist, J. O. (1979) "NIKE2D: An implicit, finite deformation, finite element cade for analyzing the static and dynamic response of two dimensional solids." Rep. UCRL-52678, Lawrence Livermore Nat. Lab., Univ. of California, Livermore, Calif.

Healy, B. E., and Dodds, R. H. (1992). "A large strain plasticity model for implicit finite element analyses." Computational Mech., 9, 95-112. Hughes, T. J. R. (1984). "Numerical implementation of constitutive models: Rate-independent deviatoric plasticity." Theoretical foundation for large-scale computations for non-linear material behavior, S. Nemat-

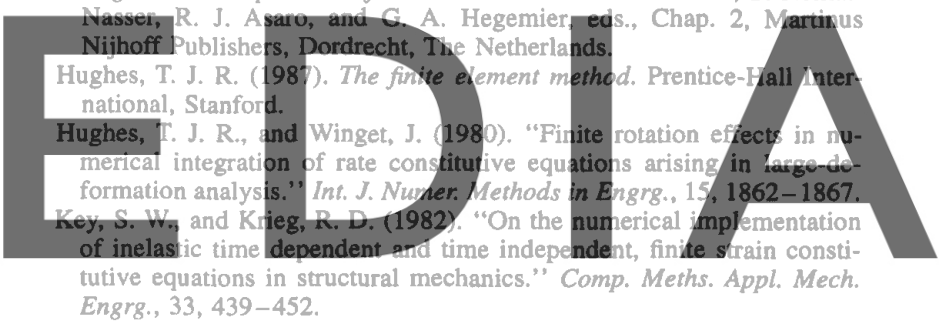

Engrg. 33, 439-452.

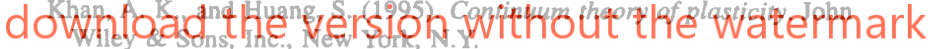

Malvern, L. W. (1969). Introduction to the mechanics of a continuous medium. Prentice-Hall Series in Engineering of the Physical Sciences, Englewood Cliffs, N...

Marsden, J. E., and Hughes, T. J. R. (1983). Mathematical foundations of elasticity. Prentice-Hall, Inc., Englewood Cliffs, N.J.

Nagtegaal, J. C., and de Jong, J. E. (1981). "Some computational aspects of elastic-plastic large strain analysis." Int. J. Numer. Methods in Engrg., 17, 15-41.

Pegon, P., and Guélin, P. (1986). "Finite strain plasticity in convected frames." Int. J. Numer. Methods in Engrg., 22, 521-545.

Pinsky, P. M., Ortiz, M., and Pister, K. S. (1983). "Numerical integration of rate constitutive equations in finite deformation analysis." Comp. Methods. Appl. Mech. Engrg., 40, 137-158.

Rashid, M. M., and Thorne, B. J. (1996). "Incremental objectivity in cyclic shearing deformations." Commun. Num. Meth. Engrg., 12, 863-871.

Rodríguez-Ferran, A., and Huerta, A. (1994). "A comparison of two objective stress rates in object-oriented codes." Monograph CIMNE No. 26. Barcelona, Spain.

Rodriguez-Ferran, A., Pegon, P., and Huerta, A. (1997). "Two stress update algorithms for large strains: Accuracy analysis and numerical implementation." Int. J. Numer. Methods in Engrg., 40, 4363-4404.

Rubinstein, R., and Atluri, S. N. (1983). "Objectivity of incremental constitutive relations over finite steps in computational finite deformation analysis." Comp. Meths. Appl. Mech. Engrg., 36, 277-290.

"Simulation of materials processing: Theory, methods and applications." (1995). Proc., 5th Int. Conf. on Numer. Methods in Industrial Forming Processes (NUMIFORM'95)

Zienkiewicz, O. C., and Taylor, R. L. (1991). The finite element method. Vols. 1 and 2, McGraw-Hill Inc., London, England. 